\title{
Effect of Wavelength-dependent Scattering on the Color Chromaticity of the LCD Backlight
}

\author{
Jin Hyuk Kwon', Hwa Joon Jun', Jin Seog Gwag ${ }^{1 *}$, and Hong Su Lee \\ ${ }^{I}$ Department of Physics, Yeungnam University, Gyeongsan 712-749, Korea \\ ${ }^{2}$ L\&F Co., Hosan-dong, Dal Seo-gu, Daegu 703-013, Korea
}

(Received March 29, 2013 : revised May 22, 2013 : accepted May 27, 2013)

\begin{abstract}
The effect of the wavelength-dependent scattering on the change of the color chromaticity in the lightguide panel of a liquid crystal display is examined. The white light emitted from the white LEDs located at the edge of the lightguide propagates in the lightguide and is scattered by the patterned dots that are placed on the bottom of the lightguide. The scattered lights that are far from the LEDs showed reddish color compared to the bluish color that is scattered from the near area from the LEDs. The color difference was between 0.01 to 0.06 depending on the resin and the thickness of the lightguide.
\end{abstract}

Keywords: Mie scattering, LCD lightguide, Color measurement

OCIS codes : (120.2040) Displays; (290.5820) Scattering measurements; (290.5870) Scattering, Rayleigh; (330.1710) Color, measurement

\section{INTRODUCTION}

A liquid crystal display (LCD) utilizes the white light emitted uniformly from the backlight unit that consists of the white LEDs, lightguide, and optical sheets [1]. The backlight plays important roles in LCDs that affects the brightness, angular luminance profile, and the image quality [2]. The poor color gamut and the poor brightness uniformity of the backlight result directly in the poor image quality and the poor brightness uniformity of the LCD, respectively [3-5]. Although the white light emitted from the white LEDs is well controlled with sufficient color gamut, the color nonuniformity occurs due to the wavelength-dependent scattering such as the Rayleigh scattering during propagation through the lightguide because there are fine scattering patterns on the bottom surface of the lightguide. As the white light that enters into the lightguide propagates it is scattered from the bottom scattering patterns, the blue light is scattered more than the red light in the near region from the white LEDs and so the chromaticity distribution tends to be bluish in the near region and reddish in the far region from the white LEDs [6]. This causes the nonuniformity of the chromaticity uniformity of the backlight. Typically, the tolerable chromaticity difference is $\Delta x, \Delta y=0.01$ that is close to the value the human eye cannot discriminate.

In this study, the chromaticity nonuniformity caused by the wavelength-dependent scattering inside the lightguide is examined in terms of the thickness and the bead size of the lightguide. Mie scattering intensity that is the solution to the Maxwell equations of the light scattering for the spherical particles is generally independent of the wavelength when the particle size is large enough. However, it reduces to the Rayleigh scattering that is strongly dependent on the wavelength when the particle diameter is less than the wavelength, as follows:

$$
I(\theta, \lambda)=I_{0}\left(\frac{1+\cos ^{2} \theta}{2 R^{2}}\right)\left(\frac{2 \pi}{\lambda}\right)^{4}\left(\frac{n^{2}-1}{n^{2}+1}\right)^{2}\left(\frac{d}{2}\right)^{6} \propto \frac{1}{\lambda^{4}},
$$

where $\theta, d$ and $n$ are the scattering angle, the particle diameter, and the refractive index of the particle [7]. $R$ is the distance between the particle and the observer. In displays the wavelength range of interest is the visible range between $400 \mathrm{~nm}$ and $700 \mathrm{~nm}$. Fig. 1 shows the Rayleigh scattering intensity in terms of the wavelength. From Fig. 1, it is known that the scattering intensity at $400 \mathrm{~nm}$ is about 10 times stronger than $700 \mathrm{~nm}$. The scattering patterns

\footnotetext{
*Corresponding author: sweat3000@ynu.ac.kr

Color versions of one or more of the figures in this paper are available online.
} 


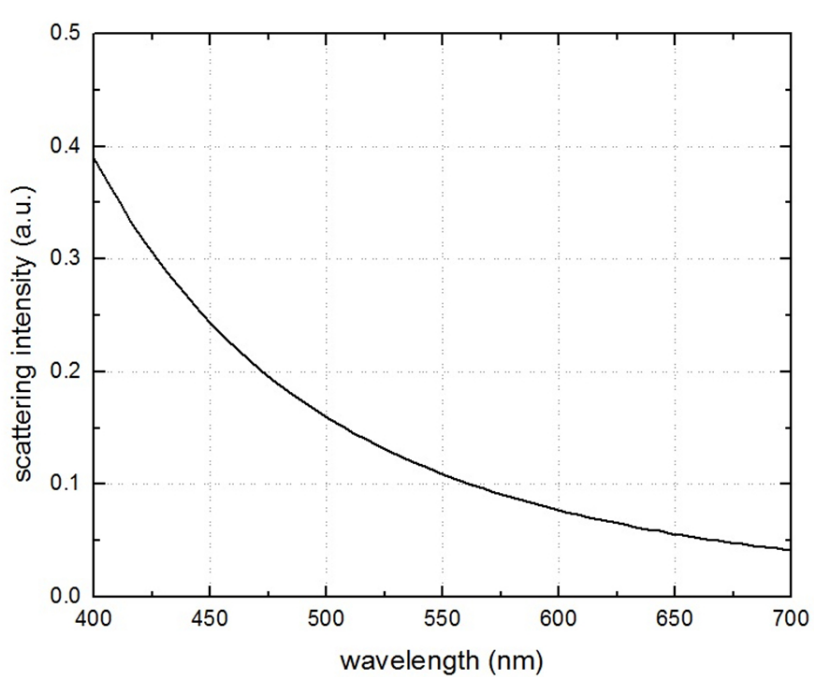

FIG. 1. The relative scattered intensity spectrum of the Rayleigh scattering in the visible range.

beneath the lightguide include transparent scattering beads of diameter around $10 \mu \mathrm{m}$ in the transparent resin. This means that the wavelength dependency of the scattering will not be so high as the Rayleigh scattering. Considering the sensitivity of human eye on the chromaticity difference, however, even small dependency of scattering intensity on the wavelength should be measured and removed.

We measured the chromaticity nonuniformity over the edge-lit LCD backlight in terms of the lightguide thickness and the bead diameter of the scattering patterns that are placed beneath the lightguide.

\section{EXPERIMENTS}

Figure 2(a) and Fig. 2(b) show the top and side views of the edge-lit LCD backlight. The size of the backlight was 50 inch diagonally. The lights from the white LEDs placed at the bottom edge enter into the lightguide and are scattered by the beads included in the scattering patterns that are placed beneath the lightguide. Three different diameters of beads of $3,7,12 \mu \mathrm{m}$ are used to scatter the light. The direction and angular width of the scattered light are controlled by the optical sheets such as the diffuser sheet and the prism sheet placed on top of the lightguide. The liquid crystal panel (LCP) is placed on top of the optical sheets. The typical measurement points are described in Fig. 1(a) and the chromaticity nonuniformity is examined along the vertical line that included the measurement points numbered as $16,8,5,2$, and 11 in Fig. 1(a). Fig. 2(b) shows the side view of the backlight. The regions $\mathrm{A}, \mathrm{B}, \mathrm{C}$ correspond to areas that are near, intermediate, and far from the white LEDs. It is expected that the near region A will show more bluish chromaticity and the far region $\mathrm{C}$ will show more reddish chromaticity

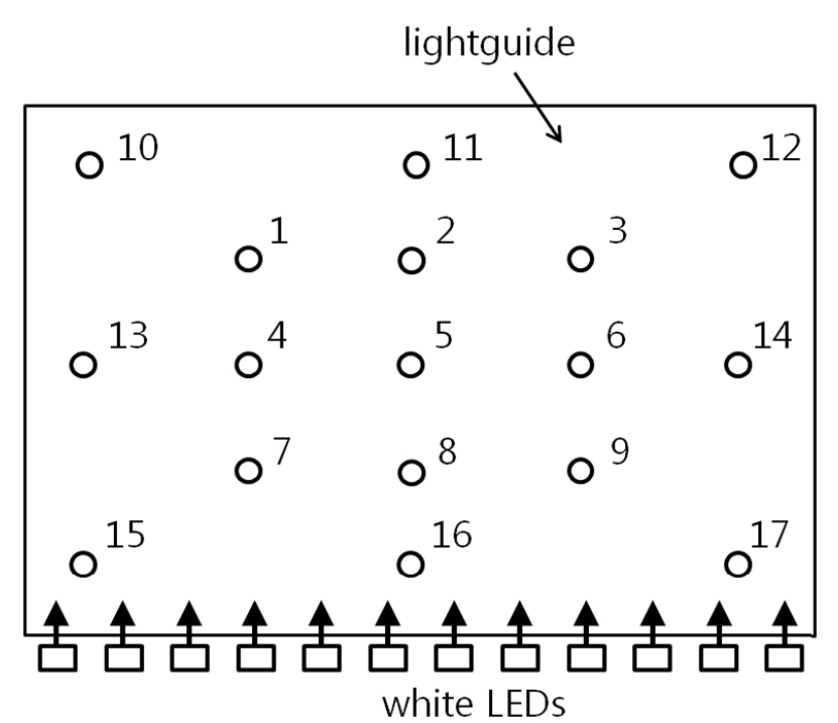

(a)

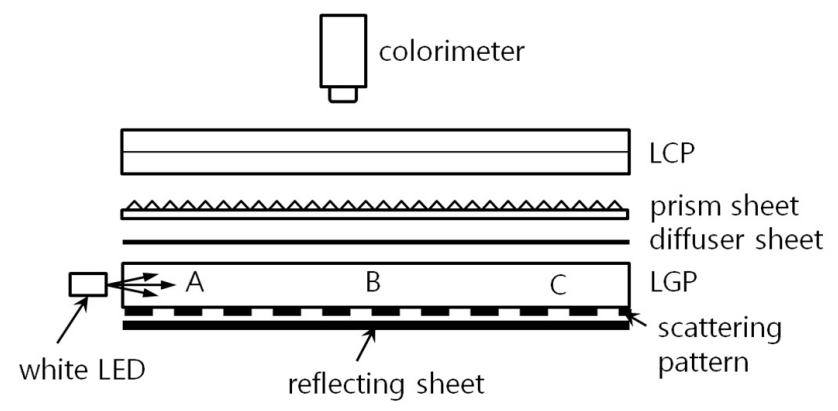

(b)

FIG. 2. (a) The top view of the LCD backlight with the measurement points, (b) the side view of the LCD backlight showing the scattering patterns on the bottom of the lightguide panel (PGP).

than the intermediate region B due to the wavelength-dependency of light scattering. Fig. 3 shows the magnified scattering patterns that include the scattering beads. The sizes of the scattering beads were 3,7 , and $12 \mu \mathrm{m}$ in diameter. The scattering beads are transparent $\mathrm{SiO}_{2}$ particles with the refractive index 1.46 at $587.6 \mathrm{~nm}$. The beads were embedded in the transparent resin PMMA (Polymethylmethacrylate) that has the refractive index of 1.49 at $587.6 \mathrm{~nm}$ to form the scattering patterns. The scattering pattern size was variable from $121 \mu \mathrm{m}$ at the entrance region to $268 \mu \mathrm{m}$ at the far region in order to generate the uniform illumination lights at the LCP. Although all of the used particle sizes are much larger than the wavelength, it is expected that the scattering beads with smaller diameter of $3 \mu \mathrm{m}$ may generate more chromaticity difference compared to the scattering beads with larger diameter of $12 \mu \mathrm{m}$ because still small wavelength dependency between the Rayleigh region and the Mie region remains. 


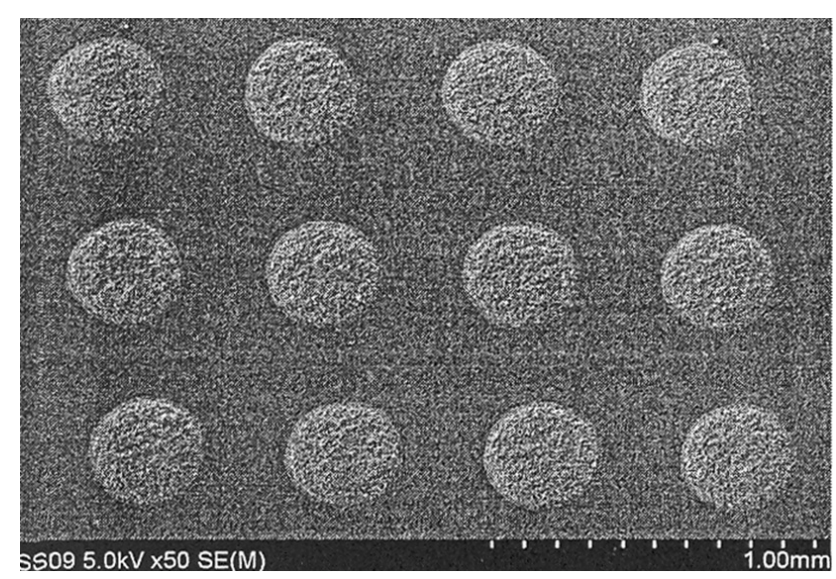

FIG. 3. The scanning electron microscopic view of the scattering patterns.

The thicknesses of the used lightguides were $0.9,1.4$, and $2 \mathrm{~mm}$. The smaller thickness create more interactions between the light and the scattering patterns, so larger chromaticity difference is expected compared to the thicker lightguide. The measurement device was the PR650 colorimeter from Photo Research. The chromaticity difference $\Delta x$ and $\Delta y$ are the chromaticity difference between the measurement points of 11 and 16 that are the measurement points near and far from the white LEDs [8]. That is,

$$
\begin{aligned}
& \Delta x=\operatorname{CIE} x(11)-\operatorname{CIE} x(16) \\
& \Delta y=\operatorname{CIE} y(11)-\operatorname{CIE} y(16),
\end{aligned}
$$

And

$$
\Delta \mathrm{W}=\sqrt{\Delta x^{2}+\Delta y^{2}} .
$$

\section{RESULTS}

Figure 4 shows the measured chromaticity difference in terms of the thickness of the lightguide with the scattering beads of diameter $7 \mu \mathrm{m}$. It is clearly shown that the chromaticity difference of $\Delta \mathrm{W}=0.0176$ in the $0.9 \mathrm{~mm}$ thick lightguide is much larger than $\Delta \mathrm{W}=0.007$ in the $2 \mathrm{~mm}$ thick lightguide. Considering the tolerable chromaticity limit of $\Delta \mathrm{W}=0.01$, that is close to the human eye limit, the lightguide of thickness $0.9 \mathrm{~mm}$ is unacceptable and the lightguide of thickness 1.4 and $2 \mathrm{~mm}$ that had the chromaticity difference 0.009 and 0.007 , respectively, are acceptable for use. Fig. 5 shows the measured chromaticity difference in terms of the scattering bead diameter in the lightguide of thickness $1.4 \mathrm{~mm}$. Even though the chromaticity difference of 0.007 when $3 \mu \mathrm{m}$ diameter beads were used is a little bit larger than the chromaticity difference of 0.0057 and 0.0058 with 7 and $12 \mu \mathrm{m}$ beads, respectively, all of them are under the tolerance limit of $\Delta \mathrm{W}=0.01$.

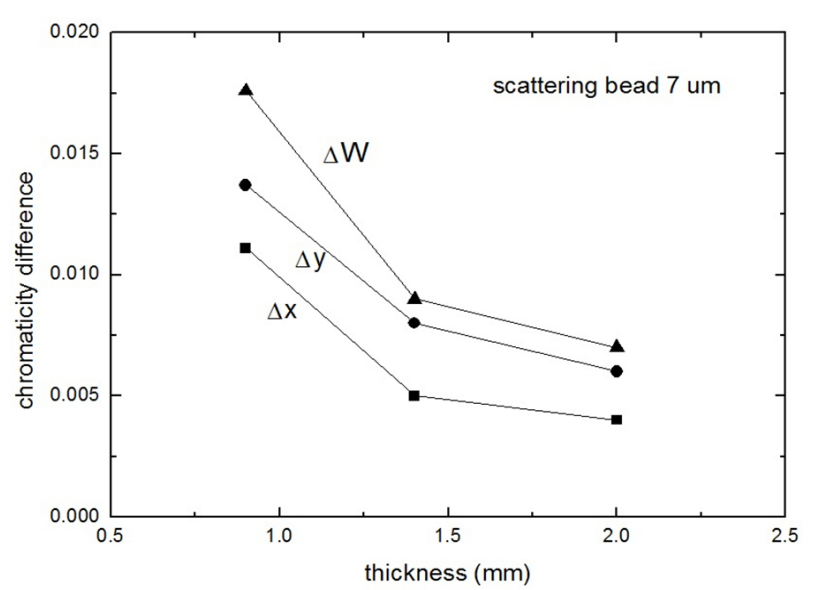

FIG. 4. The chromaticity difference in terms of the lightguide thickness.

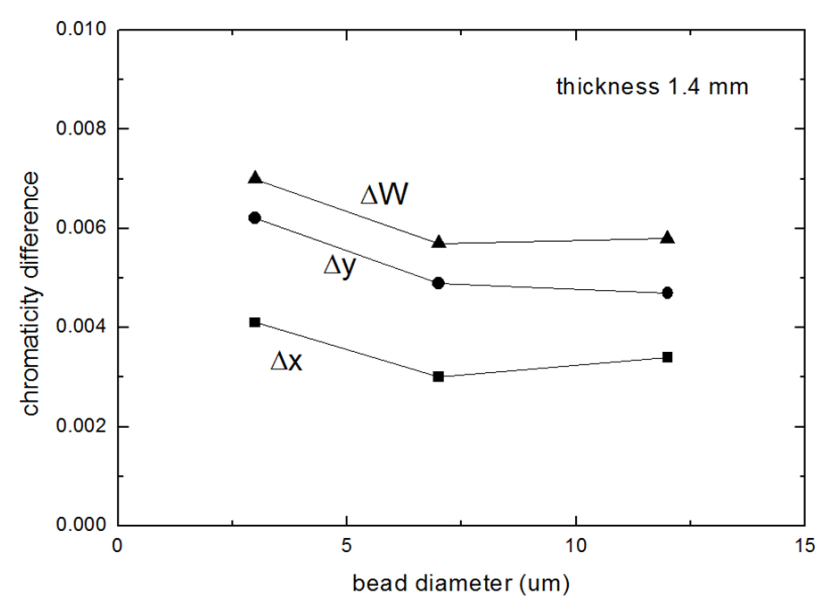

FIG. 5. The chromaticity difference in terms of the scattering bead diameters.

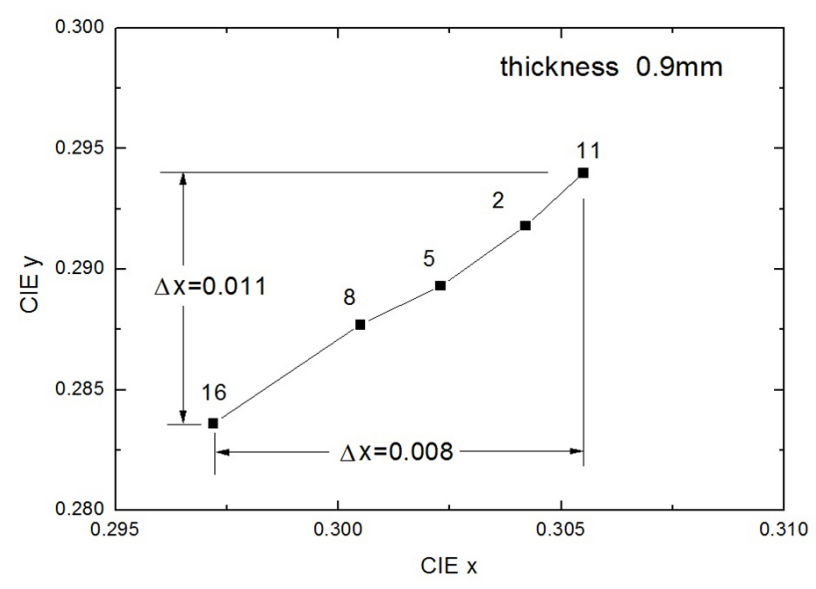

FIG. 6. The distribution of chromaticity coordinates of the five measurement points numbered as $16,8,5,2$, and 11 .

Figure 6 shows the change of chromaticity coordinates in terms of the measurement positions numbered as 16,8 , 5,2 , and 11 for the lightguide thickness $0.9 \mathrm{~mm}$. The 
measurement point 16 is the closest position from the white LED and so its chromaticity coordinate is toward the blue region and the measurement position 11 is the farthest from the white LED and its chromaticity coordinate is toward the red region. This tendency of chromaticity change shows that the chromaticity difference is induced from the wavelength-dependent scattering in the scattering patterns of the lightguide.

\section{DISCUSSION}

We showed that the chromaticity coordinates of the backlight changes from the bluish color to the reddish color when the light from the white LED propagates through the lightguide. When thinner lightguide is used the chromaticity change is more serious than the thicker lightguide because more interactions between the light and the scattering patterns occur. However, the technical trend is using thinner lightguide in order to reduce the display thickness and the cost. Also, the chromaticity difference when scattering beads larger than $7 \mu \mathrm{m}$ are used satisfies the tolerance limit. The technical issues are developing thinner backlights using fine beads and so more detailed work is needed to establish the optimum condition of the backlight size, thickness, and the bead size.

Another discussion about the chromaticity nonuniformity will be the effect of absorption in the resin and scattering beads. The used scattering beads $\left(\mathrm{SiO}_{2}\right)$ and the resin are basically well proven to be transparent enough for use in backlights. It is thought that the major effect of the color nonuniformity comes from the scattering effect because the chromaticity difference is reduced as seen Fig. 5 when the particle size is changed from $3 \mu \mathrm{m}$ to $12 \mu \mathrm{m}$ with other conditions not changed. The $\mathrm{SiO}_{2}$ beads have been widely used and proved to be transparent throughout the visible spectrum. If there were some effect from the absorption in the resin the chromaticity difference should be negligible because the thickness of the lightguide and the resin are kept same.

\section{CONCLUSION}

We measured the chromaticity nonuniformity in the 50 inch LCD backlight that is supposed to occur due to the wavelength-dependent scattering. As the backlight thickness becomes thinner than $1 \mathrm{~mm}$, the chromaticity difference became bigger than the tolerance limit. The scattering bead size used were 3,7 , and $12 \mu \mathrm{m}$ and the light scattering from particles of these sizes belong to the Mie scattering region that is almost independent of the wavelength. However, because the chromaticity tolerance limit of the human eye is very sensitive, even the small change of the chromaticity coordinate belonging to the intermediate region between the Rayleigh scattering and the Mie scattering needs to be measured and corrected. Since the chromaticity nonuniformity is strongly dependent on the backlight size, thickness, and the scattering bead size, development of the backlight need to be based on the wavelength-dependent scattering as shown in this research.

\section{ACKNOWLEDGMENT}

This research was supported by the Yeungnam University research grants in 2010.

\section{REFERENCES}

1. S. Kobayashi, S. Mikoshiba, and S. Lim, LCD Backlights (John Wiley \& Sons, West Sussex, United Kingdom, 2009).

2. K. Kakinuma, "Technology of wide color gamut backlight with light-emitting diode for liquid crystal display television," Jpn. J. Appl. Phys. 45, 4330-4334 (2006).

3. C. G. Son, J. H. Yi, J. S. Gwag, J. H. Kwon, and G. Park, "Improvement of color and luminance uniformity of the edge-lit backlight using the RGB LEDs," J. Opt. Soc. Korea 15, 272-277 (2011).

4. I. Kim and K. Chung, "Wide color gamut backlight with three band white LED," J. Opt. Soc. Korea 11, 67-70 (2007).

5. R. J. Xie, N. Hirosaki, and T. Takeda, "Wide color gamut backlight for liquid crystal displays using three-band phosphor converted white light-emitting diodes," Appl. Phys. Express 2, 022401 (2009).

6. A. Tagaya, M. Nagai, Y. Koike, and K. Yokoyama, "Thin liquid-crystal display backlight system with highly scattering optical transmission polymers," Appl. Opt. 40, 6274-6280 (2001).

7. Seinfeld and Pandis, Atmospheric Chemistry and Physics, 2nd ed. (John Wiley and Sons, New Jersey, USA, 2006), Chapter 15.

8. R. S. Berns, Principles of Color Technology (John Wiley $\&$ Sons, New York, USA, 2000). 